\title{
Correction to: The education gradient in cancer screening participation: a consistent phenomenon across Europe?
}

\author{
Barbara Willems $^{1}$ (D) Piet Bracke ${ }^{1}$
}

Published online: 23 July 2018

(C) Swiss School of Public Health (SSPH+) 2018

\section{Correction to: Int J Public Health (2018) 63:93-103 https://doi.org/10.1007/s00038-017-1045-7}

The authors would like to correct the errors in the publication of the original article. More specifically, it concerns some misclassifications of countries into organised vs. opportunistic. In the case of cervical cancer screening, Croatia had an opportunistic instead of organised screening strategy in 2006, while Denmark had an organised screening strategy instead of opportunistic screening. In the case of breast cancer screening, Germany had an organised programme instead of opportunistic screening in 2006. Lastly, with regards to colorectal cancer screening Poland had no organised programme in 2006. Due to correction of the cross-level interactions for breast and colorectal cancer screening (these changed from marginally significant to significant with the correct classification), some sentences were rephrased. Although the misclassifications did not hamper the interpretation of the results, the authors sincerely apologize for the errors. The corrected details are given below for your reading.

In the Abstract, 2nd sentence of result section should read as:

Educational inequalities in cancer screening participation were significantly smaller in countries with organised screening for cervical $(\mathrm{OR}=0.696,95 \%$ CI $0.531-0.912)$, breast $(\mathrm{OR}=0.628,95 \% \mathrm{CI} 0.438-0.900)$ and colorectal $(\mathrm{OR}=0.531,95 \%$ CI $0.303-0.932)$ cancer than they were in countries with opportunistic screening.

The original article can be found online at https:// doi.org/10.1007/s00038-017-1045-7.

Barbara Willems

Barbara.Willems@UGent.be

1 Ghent University, Ghent, Belgium
In the Results, 1st sentence of 2nd paragraph should read as:

For cervical cancer screening, overall participation ranged from $9.4 \%$ in Romania to $69.3 \%$ in Austria, with a similar overall participation rate in countries with organised $(45.1 \%)$ and opportunistic screening (49.9\%) (Table 1). Overall, breast cancer screening participation varied between $8.5 \%$ in Romania to $72.1 \%$ in Austria and France, with $12.1 \%$ (51.9-39.8\%) more participation in countries with organised screening strategies (Table 2). In comparison with cervical and breast cancer screening, participation in colorectal cancer screening was much lower, ranging from $2.5 \%$ in Sweden to $31.8 \%$ in Germany. In addition, only 4 of the 27 European countries had organised screening strategies for colorectal cancer (Table 3).

In the Results, 3rd sentence of 3rd paragraph should read as:

Table 4 provides the results of the multilevel logistic regressions. Educational inequalities in screening participation were significant for the three cancer types. Compared to the lowest educational group, the probability of an individual from the highest educational group participating in screening was 1.770 times higher for cervical cancer (95\% CI 1.540-2.034), 1.383 times higher for breast cancer (95\% CI 1.159-1.649) and 1.486 times higher for colorectal cancer (95\% CI 1.212-1.822). In addition, being employed and having a partner significantly increased the probability of participating in cervical cancer screening and breast cancer screening. The cross-level interactions indicate that educational inequalities in cancer screening participation varied significantly according to a country's screening strategy: educational inequalities were smaller in countries with organised screening strategies for cervical $(\mathrm{OR}=0.696,95 \%$ CI $0.531-0.912)$, breast $(\mathrm{OR}=0.628$, 95\% CI $0.438-0.900)$ and colorectal $(\mathrm{OR}=0.531,95 \% \mathrm{CI}$ 0.303-0.932) cancer, than they were in countries with opportunistic screening strategies. 
In the Discussion, 1st sentence of 6th paragraph should read as:

With regard to the second research question ('Do educational inequalities in cancer screening participation vary according to country-specific screening strategies?'), the results of this study clearly indicate that countries with organised cancer screening for cervical, breast and colorectal cancer allow for more equality in cancer screening participation between groups with lower and higher education than do countries with opportunistic screening.

Corrected Tables 1, 2, 3 and 4 provided here (corrected values are bold):

Table 1 Number of cases, participation rate (\%) (overall and by educational level), participation rate difference (PRD = participation tertiary - participation primary) and participation rate ratio (PRR = participation tertiary/participation primary) of cervical cancer screening in the preceding 12 months in women within the appropriate age range, by country of residence and type of cancer screening strategy. Source: Eurobarometer 66.2 (European Union 2006)

\begin{tabular}{|c|c|c|c|c|c|c|c|c|}
\hline \multicolumn{9}{|c|}{ Cervical cancer screening } \\
\hline \multirow{2}{*}{$\begin{array}{l}\text { Screening type } \\
\text { and country }\end{array}$} & \multirow[t]{2}{*}{$N$} & \multirow[t]{2}{*}{ Age range } & \multirow{2}{*}{$\begin{array}{l}\text { Overall } \\
\text { participation }(\%)\end{array}$} & \multicolumn{3}{|c|}{ Participation by educational level } & \multirow[t]{2}{*}{$\operatorname{PRD}(\%)$} & \multirow[t]{2}{*}{ PRR } \\
\hline & & & & Primary (\%) & Secondary $(\%)$ & Tertiary $(\%)$ & & \\
\hline Organised & 3735 & & 45.1 & 41.9 & 44.2 & 47.7 & 5.9 & 5.8 \\
\hline Netherlands & 304 & $30-60$ & 31.6 & 14.8 & 31.2 & 35.3 & 20.5 & 2.39 \\
\hline Denmark & 258 & 23-59 & 41.9 & 20 & 25.9 & 44.2 & 24.2 & 2.21 \\
\hline Estonia & 257 & $30-59$ & 29.2 & 30.8 & 31.7 & 25.7 & -5.1 & 0.83 \\
\hline Finland & 327 & $25-65$ & 51.7 & 30.6 & 46.2 & 58.1 & 27.5 & 1.9 \\
\hline Sweden & 297 & $23-60$ & 44.1 & 54.5 & 40.2 & 45.2 & -9.3 & 0.83 \\
\hline UK & 460 & $20-64$ & 41.7 & 37.5 & 42 & 45.2 & 7.7 & 1.21 \\
\hline Portugal & 353 & $25-64$ & 50.7 & 50.9 & 52.6 & 45.7 & -5.2 & 0.90 \\
\hline Italy & 475 & $25-64$ & 52 & 48.2 & 51.7 & 57.8 & 9.6 & 1.20 \\
\hline Slovenia & 382 & $20-64$ & 55.8 & 46.8 & 54.1 & 63.8 & 17 & 1.36 \\
\hline Lithuania & 258 & $30-60$ & 40.7 & 25 & 36.2 & 47.3 & 22.3 & 1.89 \\
\hline Hungary & 364 & $25-65$ & 46.2 & 28.9 & 52.8 & 58.5 & 29.6 & 2.02 \\
\hline Opportunistic & 6230 & & 49.9 & 37 & 50.9 & 57.7 & 20.7 & 1.56 \\
\hline Austria & 440 & $20+$ & 69.3 & 64.5 & 72 & 66.1 & 1.6 & 1.02 \\
\hline Germany & 706 & $20+$ & 54.5 & 40 & 58.2 & 66.7 & 26.7 & 1.67 \\
\hline Luxembourg & 244 & $15+$ & 66 & 59.1 & 63.9 & 74.1 & 15 & 1.25 \\
\hline France & 359 & $20-65$ & 61.8 & 48.8 & 62 & 65.1 & 16.3 & 1.33 \\
\hline Belgium & 358 & $25-64$ & 63.7 & 51.9 & 55.3 & 72.4 & 20.5 & 1.39 \\
\hline Latvia & 487 & $20-70$ & 61 & 53.5 & 58.3 & 68.5 & 15 & 1.28 \\
\hline Ireland & 343 & $25-60$ & 38.2 & 24.5 & 37.7 & 47 & 22.5 & 1.92 \\
\hline Spain & 373 & $18-65$ & 41.6 & 34.4 & 38.9 & 59.8 & 25.4 & 1.74 \\
\hline Croatia & 369 & - & 53.1 & 34.7 & 56.3 & 59.8 & 25.1 & 1.72 \\
\hline Greece & 487 & $20+$ & 46 & 29.2 & 53.8 & 68.4 & 39.2 & 2.34 \\
\hline Cyprus & 167 & $30-60$ & 49.1 & 46.7 & 52.4 & 44 & -2.7 & 0.94 \\
\hline Poland & 308 & $25-59$ & 40.6 & 26.5 & 34.8 & 51.3 & 24.8 & 1.94 \\
\hline Czech Republic & 484 & $25-69$ & 47.5 & 25.6 & 50.3 & 45.7 & 20.1 & 1.79 \\
\hline Slovakia & 502 & $23-64$ & 56 & 25 & 57.6 & 57.6 & 32.6 & 2.30 \\
\hline Romania & 318 & $25-65$ & 9.4 & 3.1 & 10.2 & 12.7 & 9.6 & 4.10 \\
\hline Bulgaria & 285 & $31-65$ & 19.6 & 8.1 & 12.7 & 34.7 & 26.6 & 4.28 \\
\hline Europe & 9965 & & 48.1 & 38.8 & 48.7 & 53 & 14.2 & 1.37 \\
\hline
\end{tabular}


Table 2 Number of cases, participation rate (\%) (overall and by educational level), participation rate difference (PRD = participation tertiary - participation primary) and participation rate ratio (PRR = participation tertiary/participation primary) for breast cancer screening in the preceding 12 months in women within the appropriate age range, by country of residence and type of cancer screening strategy. Source: Eurobarometer 66.2 (European Union 2006)

\begin{tabular}{|c|c|c|c|c|c|c|c|c|}
\hline \multicolumn{9}{|c|}{ Breast cancer screening } \\
\hline \multirow{2}{*}{$\begin{array}{l}\text { Screening type } \\
\text { and country }\end{array}$} & \multirow[t]{2}{*}{$N$} & \multirow[t]{2}{*}{ Age range } & \multirow{2}{*}{$\begin{array}{l}\text { Overall } \\
\text { participation }(\%)\end{array}$} & \multicolumn{3}{|c|}{ Participation by educational level } & \multirow[t]{2}{*}{$\operatorname{PRD}(\%)$} & \multirow[t]{2}{*}{ PRR } \\
\hline & & & & Primary $(\%)$ & Secondary $(\%)$ & Tertiary $(\%)$ & & \\
\hline Organised & 3292 & & 51.9 & 52.2 & 51.8 & 51.7 & -0.5 & 0.99 \\
\hline Germany & 237 & $50-69$ & 46.4 & 48.3 & 42.2 & 52.1 & 3.8 & 1.08 \\
\hline Luxembourg & 78 & $50-69$ & 71.8 & 87.5 & 63.9 & 66.7 & -20.8 & 0.76 \\
\hline France & 154 & $50-74$ & 72.1 & 76.4 & 65.2 & 78.8 & 2.4 & 1.03 \\
\hline Belgium & 148 & $50-69$ & 66.2 & 60 & 66.2 & 69.8 & 9.8 & 1.16 \\
\hline Netherlands & 200 & $50-75$ & 60.5 & 59.6 & 52.3 & 73.7 & 14.1 & 1.24 \\
\hline Denmark & 161 & $50-69$ & 21.1 & 8.3 & 21.7 & 22.2 & 13.9 & 2.67 \\
\hline Estonia & 102 & $50-59$ & 53.9 & 40 & 52.8 & 59 & 19 & 1.48 \\
\hline Finland & 185 & $50-69$ & 54.6 & 61.5 & 57.8 & 48.8 & -12.7 & 0.79 \\
\hline Sweden & 277 & $40-74$ & 55.2 & 38.5 & 52.4 & 59.3 & 20.8 & 1.54 \\
\hline UK & 218 & $50-70$ & 40.4 & 39.6 & 42.1 & 37.5 & -2.1 & 0.95 \\
\hline Portugal & 229 & $45-69$ & 69 & 69.3 & 72 & 60 & -9.3 & 0.87 \\
\hline Spain & 188 & $45-70$ & 46.3 & 43 & 43.6 & 71.4 & 28.4 & 1.66 \\
\hline Italy & 152 & $50-69$ & 62.5 & 61.4 & 60.3 & 70.8 & 9.4 & 1.15 \\
\hline Croatia & 171 & $50-69$ & 41.5 & 34.8 & 44.9 & 47.2 & 12.4 & 1.36 \\
\hline Cyprus & 108 & $50-69$ & 44.4 & 41.3 & 47.5 & 60 & 18.7 & 1.45 \\
\hline Lithuania & 197 & $50-69$ & 23.4 & 20.6 & 22.1 & 26 & 5.4 & 1.26 \\
\hline Czech Republic & 281 & $45-69$ & 53.4 & 42.1 & 54.1 & 59.6 & 17.5 & 1.42 \\
\hline Hungary & 206 & $45-65$ & 61.7 & 51.3 & 71 & 57.5 & 6.2 & 1.12 \\
\hline Opportunistic & 2035 & & 39.8 & 29.7 & 43.1 & 44.5 & 14.8 & 1.5 \\
\hline Austria & 240 & $40-69$ & 72.1 & 63.6 & 76.1 & 71.9 & 8.3 & 1.13 \\
\hline Latvia & 171 & $50-69$ & 38 & 37.9 & 34.7 & 44.7 & 6.8 & 1.18 \\
\hline Ireland & 119 & $50-64$ & 44.5 & 51.5 & 42 & 41.2 & -10.3 & 0.80 \\
\hline Slovenia & 200 & $50-69$ & 37 & 29.8 & 39.4 & 40.8 & 11 & 1.37 \\
\hline Greece & 195 & $40-64$ & 45.6 & 33.7 & 51.6 & 65.8 & 32.1 & 1.95 \\
\hline Poland & 160 & $50-69$ & 39.4 & 24.4 & 40 & 53.3 & 28.9 & 2.18 \\
\hline Slovakia & 453 & $40+$ & 49.7 & 36.4 & 52.9 & 48 & 11.6 & 1.32 \\
\hline Romania & 164 & $50-69$ & 8.5 & 4 & 1.8 & 29.4 & 25.4 & 7.35 \\
\hline Bulgaria & 333 & $40+$ & 15.9 & 9 & 13.6 & 26.7 & 17.7 & 2.97 \\
\hline Europe & 5327 & & 47.3 & 44.7 & 47.9 & 49.4 & 4.7 & 1.11 \\
\hline
\end{tabular}


Table 3 Number of cases, participation rate (\%) (overall and by educational level), participation rate difference (PRD = participation tertiary - participation primary) and participation rate ratio ( $P R R=$ participation tertiary/participation primary) for colorectal cancer screening in the preceding 12 months in men and women within the appropriate age range, by country of residence and type of cancer screening strategy. Source: Eurobarometer 66.2 (European Union 2006)

\begin{tabular}{|c|c|c|c|c|c|c|c|c|}
\hline \multicolumn{9}{|c|}{ Colorectal cancer screening } \\
\hline \multirow{2}{*}{$\begin{array}{l}\text { Screening type } \\
\text { and country }\end{array}$} & \multirow[t]{2}{*}{$N$} & \multirow[t]{2}{*}{ Age range } & \multirow{2}{*}{$\begin{array}{l}\text { Overall } \\
\text { participation } \\
(\%)\end{array}$} & \multicolumn{3}{|c|}{ Participation by educational level } & \multirow[t]{2}{*}{$\operatorname{PRD}(\%)$} & \multirow[t]{2}{*}{ PRR } \\
\hline & & & & Primary (\%) & Secondary $(\%)$ & Tertiary $(\%)$ & & \\
\hline Organised & 1937 & & 7.7 & 8.1 & 7.4 & 7.5 & -0.6 & 0.93 \\
\hline Finland & 183 & $60-69$ & 11.5 & 19.6 & 12.1 & 5.6 & -14 & 0.29 \\
\hline UK & 575 & $45-74$ & 5.6 & 7 & 5.1 & 3.2 & -3.8 & 0.46 \\
\hline Italy & 278 & $50-74$ & 8.6 & 9.4 & 4.9 & 15.4 & 6 & 1.64 \\
\hline Czech Republic & 446 & $50+$ & 9 & 11.9 & 9.2 & 5.6 & -6.3 & 0.47 \\
\hline Opportunistic & 7706 & & 10.3 & 9.8 & 10.1 & 11.2 & 1.4 & 1.14 \\
\hline Austria & 372 & $50+$ & 27.2 & 19.7 & 29.3 & 38.8 & 19.1 & 1.97 \\
\hline Germany & 592 & $50-74$ & 31.8 & 28.9 & 31.8 & 37 & 8.1 & 1.28 \\
\hline Luxembourg & 172 & - & 19.2 & 17 & 22.2 & 17 & 0 & 1 \\
\hline France & 317 & $50-74$ & 16.1 & 18.6 & 14.3 & 16.3 & -2.3 & 0.88 \\
\hline Belgium & 353 & $50-75$ & 10.5 & 13.1 & 9.9 & 9.4 & -3.7 & 0.72 \\
\hline Netherlands & 298 & $55-75$ & 4.7 & 1.3 & 3.3 & 9.4 & 8.1 & 7.23 \\
\hline Denmark & 459 & $45-75$ & 8.3 & 6.3 & 8.3 & 8.4 & 2.1 & 1.33 \\
\hline Latvia & 327 & $50-74$ & 16.5 & 18 & 9.4 & 30.6 & 12.6 & 1.7 \\
\hline Estonia & 389 & $50-74$ & 3.3 & 1.6 & 4.6 & 2.3 & 0.7 & 1.44 \\
\hline Sweden & 201 & $50-60$ & 2.5 & 0 & 3.4 & 2.5 & 2.5 & - \\
\hline Ireland & 224 & $55-74$ & 10.3 & 6.7 & 10.1 & 20 & 13.3 & 2.99 \\
\hline Portugal & 297 & $50-70$ & 12.8 & 11.5 & 17.9 & 20 & 8.5 & 1.74 \\
\hline Spain & 215 & $50-69$ & 7 & 5.1 & 7.5 & 22.2 & 17.1 & 4.35 \\
\hline Slovenia & 313 & $50-69$ & 3.5 & 6.4 & 2.1 & 2.7 & -3.7 & 0.42 \\
\hline Croatia & 335 & $50-74$ & 3.6 & 7 & 2.2 & 1.2 & -5.8 & 0.17 \\
\hline Greece & 455 & $50+$ & 7 & 5.9 & 6.7 & 13.3 & 7.4 & 2.25 \\
\hline Cyprus & 260 & $50+$ & 3.1 & 3.8 & 2.7 & 0 & -3.8 & 0 \\
\hline Lithuania & 384 & - & 7.3 & 3.2 & 7.5 & 10 & 6.8 & 3.13 \\
\hline Poland & 217 & $50-65$ & 6.9 & 3.8 & 6.7 & 10 & 6.2 & 2.63 \\
\hline Slovakia & 491 & $50+$ & 9.4 & 3.9 & 10.2 & 11.1 & 7.2 & 2.85 \\
\hline Hungary & 365 & $50-70$ & 4.9 & 7.6 & 3.8 & 2.1 & -5.5 & 0.28 \\
\hline Romania & 353 & $50-74$ & 3.1 & 3.7 & 2.1 & 4.2 & 0.5 & 1.14 \\
\hline Bulgaria & 772 & $31+$ & 4.4 & 0.6 & 5.2 & 6 & 5.4 & 10 \\
\hline Europe & 9643 & & 9.8 & 9.4 & 9.5 & 10.7 & 1.3 & 1.14 \\
\hline
\end{tabular}




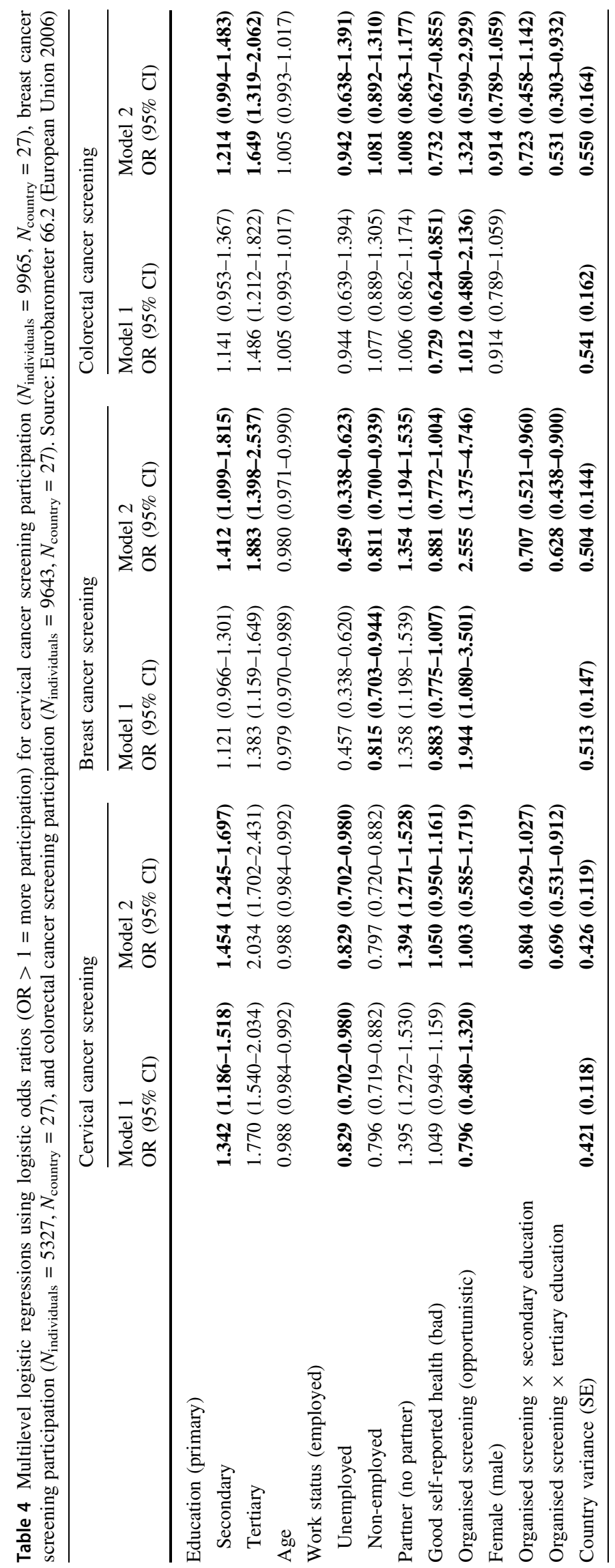


Correction to the references

Kaminski MF, Kraszewska E, Rupinski M, Laskowska M, Wieszczy P, Regula J (2015) Design of the Polish Colonoscopy Screening Program: a randomized health services study. Endoscopy 47(12):1144-1150

Lynge E, Andersen B, Christensen J, Ejersbo D, Jochumsen K, Johansen $\mathrm{T}$ et al (2017) Cervical screening in Denmark - a success followed by stagnation. Acta Oncol $1-8$

Malek D, Kääb-Sanyal V (2016) Implementation of the German Mammography Screening Program (German
MSP) and first results for initial examinations, 2005-2009. Breast Care 11(3):183-187

Petry KU, Menton S, Menton M, van Loenen-Frosch F, de Carvalho Gomes H, Holz B et al (2003) Inclusion of HPV testing in routine cervical cancer screening for women above 29 years in Germany: results for 8466 patients. Br $\mathrm{J}$ Cancer 88:1570. https://doi.org/10.1038/sj.bjc.6600918

Vrdoljak-Mozetič D, Verša Ostojić D, Štemberger-Papić S, Janković S, Glibotić-Kresina H, Brnčić-Fischer A, BenićSalamon K (2010) Cervical Cancer Screening Programme in Primorsko-Goranska County, Croatia-the results of the pilot study. Coll Antropol 34(1):225-232 